\title{
Tuberculosis of the wrist masquerading as complex regional pain syndrome type 1: A diagnostic dilemma
}

\author{
Dharmshaktu GS ${ }^{1}$, Roy AV \\ ${ }^{1}$ Dr Ganesh Singh Dharmshaktu, Assistant Professor, ${ }^{2}$ Dr Anshuman Vijay Roy, Assistant Professor . Both are affiliated \\ with Department of Orthopedics, Government Medical College, Haldwani, Uttarakhand, India
}

Address for correspondence: Dr. Ganesh Singh Dharmshaktu, E mail: drganeshortho@gmail.com

\begin{abstract}
Tuberculosis of the wrist region is unusual site and requires high index of suspicion to get a validated diagnosis. The pattern of clinical features and their vagueness contribute to its neglect or misdiagnosis. Various other chronic pain disorders of the wrist can mimic the tubercular affectation of the wrist and vice versa. Complex regional pain syndrome type 1 is the clinical entity that can closely resemble prodromal features of tubercular disease especially in the settings of history of minor trauma and history of consequent immobilization as its treatment.
\end{abstract}

Key words: Reflex Sympathetic Dystrophy, Tuberculosis, Osteoarticular, Complex Regional Pain Syndrome Type 1

\section{Introduction}

Complex Regional Pain Syndrome (CRPS) also called reflex sympathetic dystrophy in the past is a multifaceted chronic neuropathic pain disorder. Owing to varied patterns of morbidity, it is neither managed easily nor there are good evidence based guidelines [1,2].

It affects 25.2 cases per 100,000 as described based on criteria laid down by International Association for the Study of Pain (IASP) [3, 4].

Most of the time a triggering event is noted and sprains and fractures are the commonest of all [5]. CRPS type 1 differs from type 2 as the former has absence of associated nerve injuries. Literature states that proper history and clinical examination is the cornerstone of its diagnosis.

\section{Case Report}

The case was a 46 year lady who had history of preexisting off and on right side wrist pain for last five months. She was taking occasional pain medication for the pain with transient effect.

She suffered a low energy trauma with the mechanism of fall on the outstretched hand three days before she consulted us in view of pain, swelling and difficulty in wrist and hand functions thus affecting her activities of daily living.
Radiograph of the case revealed no obvious or occult fracture and any significant deformity. She was advised a plaster of paris below elbow slab in order to alleviate pain and swelling for one or two weeks. She was compliant to the treatment but pain and swelling was mildly reduced thus the splintage continued for another week.

After that she had a ten day course of physiotherapy in view of finger and wrist stiffness. She was on pain medications on as and when required basis along with calcium and vitamin D supplements.

The pain score improved but not completely abolished and it was significant on active or passive finger and wrist movements. The patient had a feeling of clumsiness when initiating any activity with that extremity.

The swelling was also not completely resolved and the most troublesome element was the stiffness in the wrist. No other associated disorders and 'red flags' were found. The radiograph of the wrist at one and half month interval showed spotty osteoporosis with hazyness of bony architecture [Fig 1].

A provisional diagnosis of complex regional pain syndrome was made and patient was advised supervised physiotherapy and oral bisphosphonates along with calcium supplementation. Recalcitrant complaints were analyzed with regional MRI. [Fig 2 \& 3] 


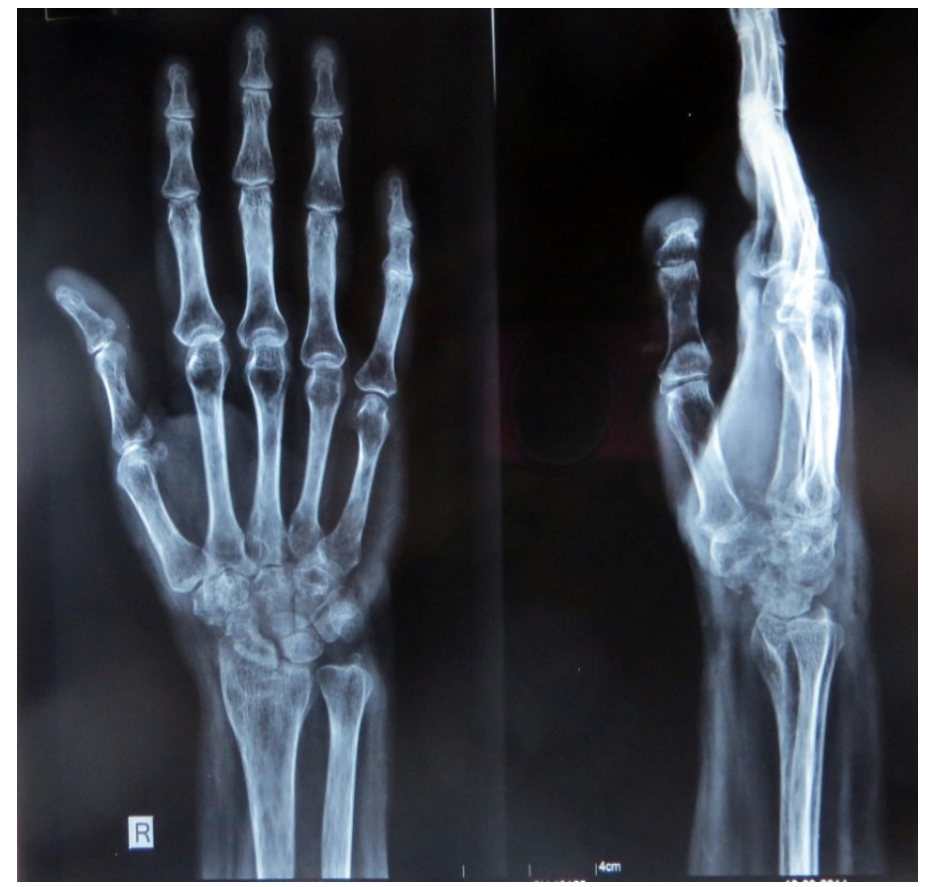

Fig 1: Radiograph showing features of CRPS 1 following trauma.

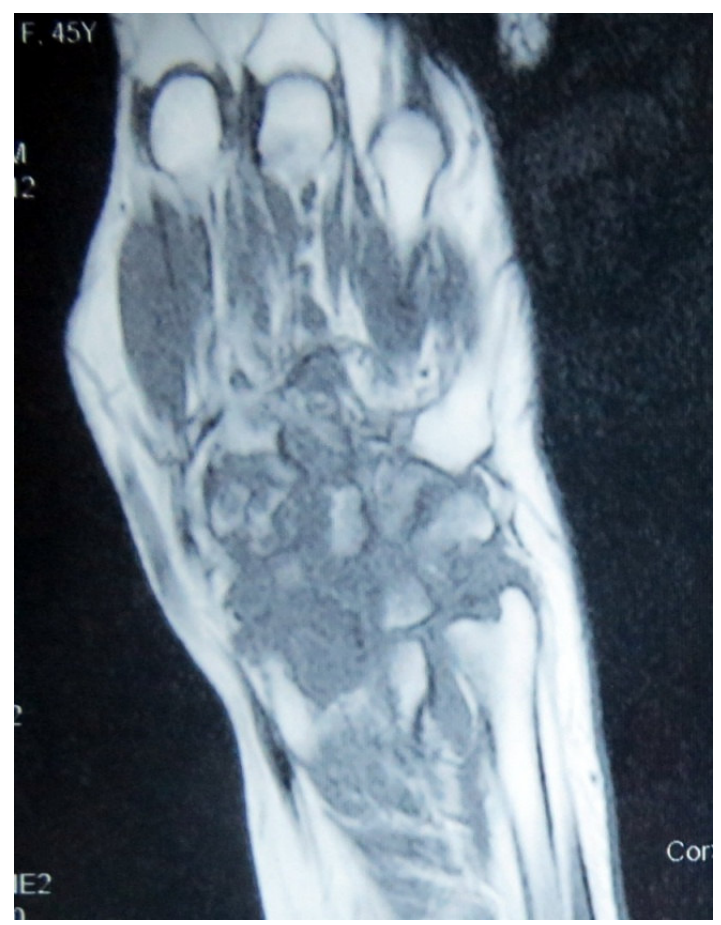

Fig 2: MRI showing wrist bones and adjacent soft tissue pathology. 


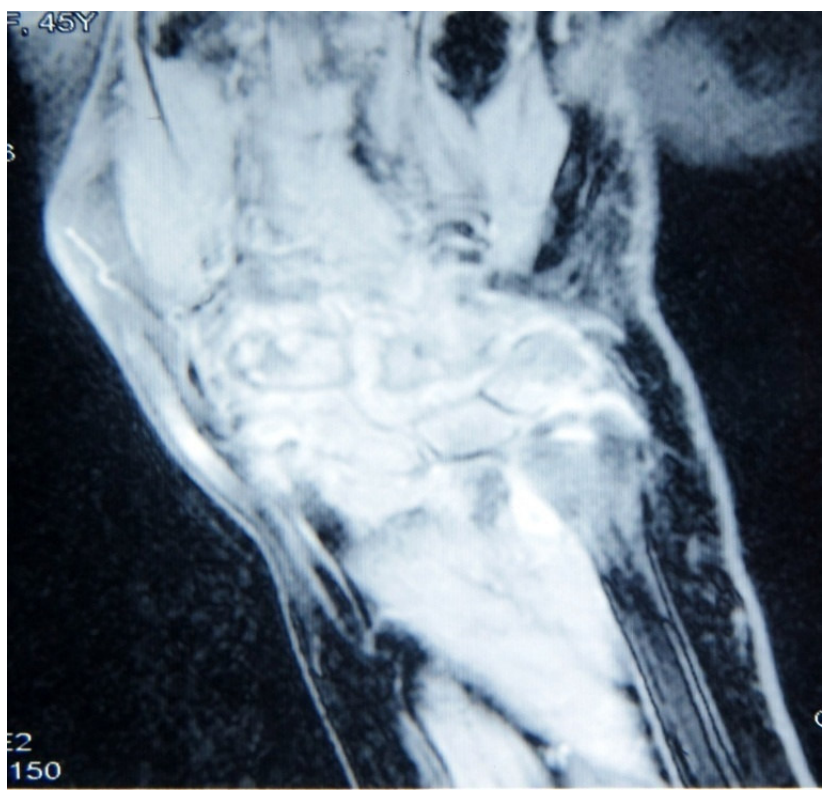

Case Report

Fig 3: MRI showing inflammatory edema with tubercular etiology.

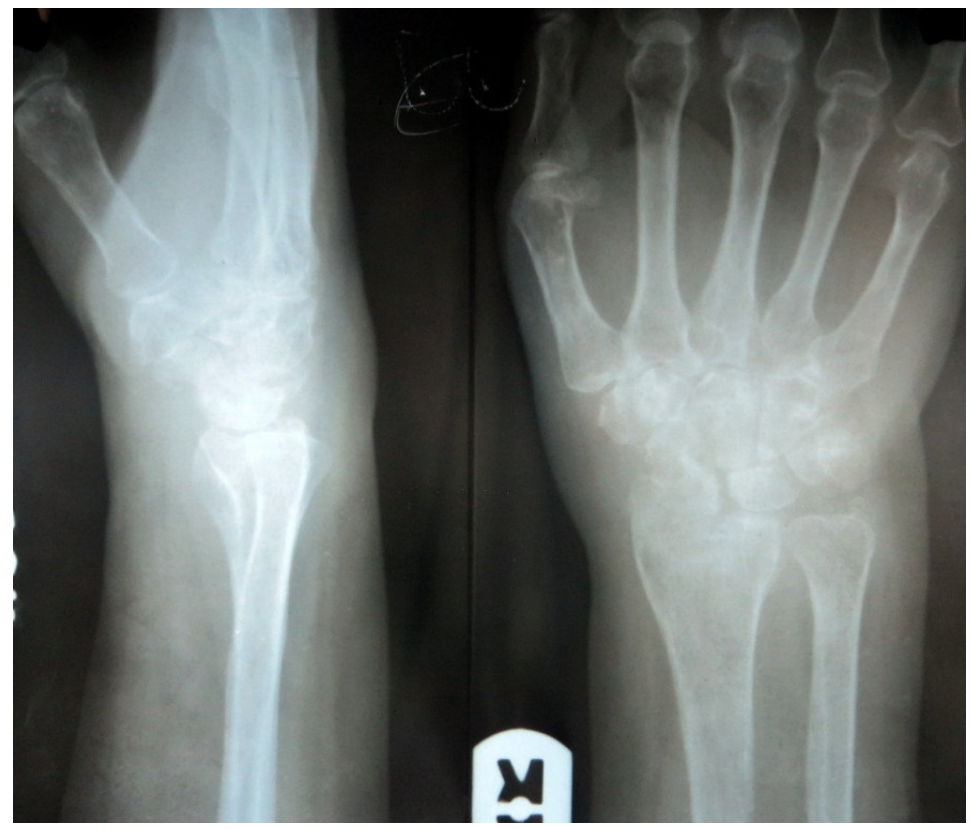

Fig 4: Six months after anti-tubercular therapy, improvement in bone radiography.

MRI showed marked erosion and large edema involving distal radius, ulna, multiple carpals and base of second and third metacarpals. There was marked synovial thickening and fluid seen along distal radio-ulnar, radiocarpal, inter-carpal and few carpo-metacarpal joints.

Marked fluid and synovial thickening was seen along flexor and extensor tendon sheaths. Rest of the study was unremarkable and impression was of an infective or inflammatory arthritis with probable tubercular etiology.

\section{Result}

The patient was put on four drugs anti tubercular treatment and she showed good clinical response and improvement in all parameters when reviewed a month later. A follow up of three, six and twelve and final 
eighteen months were encouraging with dramatic improvement in clinical as well as radiological studies.

\section{Discussion}

Complex regional pain syndrome (CRPS) is divided into two types, based on associated neurological involvement i.e. type 1 without and type 2 with neurological features. The common precipitating factor is traumatic events often with variable period of immobilization and splintage as its treatment.

The classical features include pain, stiffness of wrist, fingers and sometimes other joints [6]. Apart from shiny skin with loss of sweat and radiological features like patchy osteoporosis, vasomotor changes can be found in variable severity. The condition has predilection for female sex, middle age and distal part of extremities like hand and foot [7].

Most of the features of tuberculosis can be overlooked by this presentation. Thus in the settings of CRPS, a good follow-up and use of advance imaging modalities like magnetic resonance imaging (MRI) can help differentiate between the two for better management.

Tubercular infection of the wrist region mimicking CRPS in the setting of minor soft tissue injury and consequent immobilization offers a diagnostic dilemma. Authors could find similar case reports only once as per the literature search in this regard [8].

\section{Conclusion}

The constellation of clinical features may at times mask the underlying disorder other than CRPS, more notably in cases where minor traumatic injuries and immobilization is also associated. A meticulous attention to detail about auxillary pointers and taking help of modern imaging modalities like MRI can be instrumental to better management of such cases.

\section{References}

1. Tran de QH,Duong S,Bertini P,Finlayson RJ : Treatment of complex regional pain syndrome : A review of the evidence. Can J Anaesth 2010;57(2): 146-166.

2. Mackey S,Feinberg S: Pharmacologic therapies for complex regional pain syndrome. Curr Pain Headache Rep 2007;11(1):38-43.

3. demos M,de Bruijn AG,Huygen FJ,Dielman JP ,Stricker BH, Sturkenboom MC : The incidence of complex regional pain syndrome:A population- based study.Pain 2007; 129(1-2):12-20.

4. Merskey H,Bogduk N: Classification of Chronic Pain: Description of Chronic Pain Syndromes and Definitions of Pain Terms, ed 2.Seattle,WA,IASP Press,1994.

5. Harden N,Bruehl S: Complex regional pain syndrome, in Fishman SM,Ballantyne JC, Rathmell JP, eds: Bonica's Management of Pain, ed 4.Philadelphia,PA,Lippincott Williams \& Wilkins,2010, pp 314-331.

6. Scadding JW: Complex Regional Pain Syndrome. In Wall and Melzac's textbook of pain. $5^{\text {th }}$ edition. Edited by McMahon SB,Koltzenberg M.Philadelphia: Elsevier/Churchill Livingstone; 2005:835-849.

7. Tietzen R: Reflex sympathetic dystrophy of the knee. Clin Orthop Relat Res 1986,209:234-243.

8. Hakan Gundes, Bilgehan Tosun, Sibel Gundes, Alptekin Tosun, Bahar Muezzinoglu : Persistent posttraumatic wrist pain: complex regional pain syndrome? Mycobacterium tuberculosis infection should be in the differential diagnosis. European Journal of Orthopaedic Surgery \& Traumatology.2007;17(2):193197.

\section{How to cite this article?}

Dharmshaktu GS, Roy AV. Tuberculosis of the wrist masquerading as complex regional pain syndrome type 1: A diagnostic dilemma. Int J Med Res Rev 2014;2(3):270-273. doi:10.17511/ijmrr.2014.i03.19 